\title{
INTEGRATED CARE Older people with hip fracture transferred to intermediate care: outcomes in an integrated health and social care model
}

\author{
Authors: Una Clancy, ${ }^{\mathrm{A}}$ Mark Brown, ${ }^{\mathrm{B}}$ Ziad Alio, ${ }^{\mathrm{C}}$ Kate Wardle ${ }^{\mathrm{D}}$ and Neil Pendleton ${ }^{\mathrm{E}}$
}

Following surgery for hip fracture almost a quarter of patients do not return directly to their usual residence, using the resources within intermediate care and enablement. This was a retrospective cohort study involving 156 Salford residents admitted with hip fracture in 2015. Linked health data were collected on those discharged to intermediate care vs home in terms of readmissions, mortality, lengths of stay, delayed transfers of care, diagnoses of delirium and pre-existing forms of dementia. The median duration of the continuous care episode in the intermediate care cohort, inclusive of readmissions to hospital, was $\mathbf{5 2}$ days. There was a $26 \%(n=20)$ readmission rate from intermediate care. Readmission rates at 120 days were higher among those discharged to intermediate care vs home (OR 3.21, 95\% CI 1.37-7.54, $\mathrm{p}=0.007$ ) and among those with a form of dementia (OR 4.76, 95\% CI 1.79-12.63, p=0.0017). Patients with delirium during their acute admission were more likely to be discharged to intermediate care (OR 5.43, 95\% CI 2.36-12.47, p=0.0001) and were less likely to ultimately be discharged home (OR 6.40, 95\% CI 2.25-18.21, $p=0.0005$ ), as were those with some form of dementia (OR 6.60, 95\% CI 1.97-22.08, $p=0.002$ ).

Measurement of the entire care episode demonstrates significant lengths of stay. Medium term readmission rates are higher in those discharged to intermediate care. Delirium and dementia are associated with higher readmission rates and lower rates of discharge to own home. It is imperative that a whole pathway approach to commissioning hip fracture services is established.

KEYWORDS: Rehabilitation, hip fracture, cognition, episode of care, patient discharge, older people

Authors: ${ }^{\text {A }}$ specialist registrar in geriatric medicine, Western General Hospital, Edinburgh, UK; ${ }^{B}$ advanced nurse practitioner in orthogeriatrics, Salford Royal Foundation Trust, Salford, UK; ' ${ }^{C}$ consultant orthogeriatrician, Salford Royal Foundation Trust, Salford, UK; D consultant orthogeriatrician, Salford Royal Foundation Trust, Salford, UK; ' professor of medical gerontology, Division of Neuroscience and Experimental Psychology, School of Biological Sciences, Manchester Academic Health Science Centre, University of Manchester Salford Royal Hospital, Manchester, UK

\section{Introduction}

Almost 65,000 people presented to hospital in the UK with a hip fracture in 2015. The annual cost for hip fracture cases in the UK, including medical and social care, is approximately $€ 2$ billion. $^{2}$ In $2014,22.3 \%$ of these patients who were admitted from their own home / sheltered housing attended bedded intermediate care (IMC) following their acute hospital admission to meet their rehabilitation need. ${ }^{3}$

The National Hip Fracture Database (NHFD) annual report identifies that there is little understanding of what happens to patients who are transferred to bedded IMC after a hip fracture. In 2016, one of the report's key recommendations was to consider a whole pathway approach to commissioning hip fracture services, including the extension of clinical governance beyond the acute part of the pathway into intermediate care. Moreover, the report recommends that 120 day follow up should be an integral part of patient care. ${ }^{1}$

At present, only part of the length of stay (LOS) in NHS-funded IMC beds is consistently captured. ${ }^{1}$ Defining and measuring the overall LOS, inclusive of readmission to hospital, is essential practice for local commissioners in planning efficient services around a growing older population. ${ }^{4}$ While it is known that the average delay in transfer of care of general hospital inpatients to bedded intermediate care is 3 days, specific data pertaining to hip fracture patients have not been published. ${ }^{5}$

Almost one-fifth of patients are estimated to have a type of dementia when they present with a hip fracture. ${ }^{6}$ Patients with dementia are known to have higher mortality and poorer functional recovery. ${ }^{7-10}$ Notwithstanding this, this cohort has been shown to be less likely to access rehabilitation following hip fracture. $^{11-14}$

Delirium occurs in $35-65 \%$ of patients admitted with hip fracture. ${ }^{15}$ Patients with delirium in the perioperative period have also been shown to have poorer functional recovery, higher rates of institutionalisation and higher mortality rates. ${ }^{16-19}$

The relationship of diagnoses of delirium and dementia with admission rates to intermediate care, readmission rates to hospital and final discharge destination, have not heretofore been studied in a UK hip fracture population.

In this study, the primary outcome measures were to compare the following in patients who were discharged to bedded intermediate care vs those who were discharged home: acute LOS, 
final discharge destination, 30- and 120-day readmission rates, 30- and 120-day mortality.

The secondary outcome measures were to assess the following: delayed transfers of care to bedded intermediate care, prevalence of inpatient diagnoses of delirium, pre-existing diagnoses of some form of dementia and relationship of these diagnoses to outcomes including transfer to bedded intermediate care, final discharge destination, readmission rates and mortality. This study also aimed to quantify the intermediate care LOS inclusive of readmission to hospital.

\section{Methods}

\section{Ethical issues}

This was a retrospective study for which formal approval was not required. Data were anonymised before any staff without direct patient contact were involved.

\section{Setting}

Salford Royal Foundation Trust (UK) is a major university teaching trust and integrated provider of hospital, community and primary care services, serving the City of Salford locally and providing specialist services to Greater Manchester and beyond. Patients with fractured neck of femur who are normally resident in Salford have access locally to three local bedded intermediate care facilities.

The rehabilitation facilities are supported by a twice-weekly visit from a community geriatrician, daily presence of an advanced nurse practitioner staff and advice from local GPs. Those who are deemed suitable for discharge directly home have access to early supported discharge (ESD) and/or packages of social care. The ESD pathway is available for those patients who are medically stable in the postoperative period, who are mobilising on the ward with a stick or unaided, and who are identified as having ongoing rehabilitation needs. Patients who were normally resident in the neighbouring Trafford region were not included in this study as we could not capture the relevant data due to differences in health record systems there.

All patients over the age of 60 admitted with a fractured neck of femur are admitted to an orthopaedic ward and have orthogeriatric input under a routine orthogeriatric review model, served by 2.4 whole time equivalent consultant orthogeriatricians during the period Monday to Friday each week.

We used local NHFD data to identify patients with fractured neck of femur from 1 January - 31 December 2015. All Salford residents who were discharged to intermediate care or to their own home / sheltered housing were identified.

The following exclusion criteria were applied: non-Salford residents, those who did not survive their acute inpatient stay, and those who were discharged directly to 24 -hour care following their acute inpatient admission.

The Electronic Patient Record (EPR) in Salford contains continuous health data spanning the acute admission through to intermediate care. Primary care and community geriatrics teams deliver healthcare to all of the IMC patients, which means that this record fully captures the patient journey until return to home. The EPR was used to identify readmission and mortality rates at 30 and 120 days.

In relation to those transferred to intermediate care following their acute admission, additional information was recorded including: number and duration of delayed transfers of care, intermediate care LOS; length of 'super-spell' ie time from acute admission to final discharge from intermediate care, readmissions from intermediate care to hospital (including number and length of readmissions), relationship between delirium or dementia and transfer to bedded intermediate care and finally, relationship between delirium or dementia and final discharge destination following the intermediate care episode.

\section{Definitions}

Where patients were readmitted from IMC to hospital and discharged back to IMC, their IMC LOS is classified as time between IMC admission date to final IMC discharge date. Where patients were readmitted from IMC to hospital and subsequently discharged to a location other than IMC (died, 24-hour care, home), their IMC LOS is classified as the time between IMC admission date to date of readmission to hospital. Previous dementia was defined as a preadmission formal diagnosis of dementia by an old age psychiatrist. Previously undiagnosed dementia was not investigated in this study.

Inpatient clinical notes on EPR were reviewed to identify acute inpatient delirium and pre-existing dementia diagnoses. Assessment for incident delirium is conducted daily on ward rounds during 5 working days. Delirium was diagnosed using a combination of the Confusion Assessment Method daily and the 4AT postoperatively. Inpatient clinical notes were retrospectively reviewed by the primary author to identify the estimated date of being classed as 'medically fit for discharge' in order to determine delayed transfers of care.

\section{Statistical analysis}

Statistical analysis was carried out using SPSS Statistics version 22. Investigations into the normality of continuous variable distributions found that they did not display a normal curve and they were therefore summarised using median and interquartile ranges (IQR), and analysed using non-parametric tests. In testing associations for categorical variables, Pearson's chi-square tests for independence and risk ratios were performed. For some analyses, a split file function was used to separately assess patients who were and were not discharged to intermediate care. A probability value of $<0.05$ was considered statistically significant.

\section{Results}

\section{Baseline characteristics}

A total of 323 patients over the age of 60 were admitted with fractured neck of femur in 2015. Of the 156 Salford residents who survived to discharge and were not discharged to 24 -hour care, $51 \%$ (80) were discharged home and 49\% (76) were transferred to bedded intermediate care following their acute admission. The median age was 80 years (SD 8.9) and $71.2 \%(n=111)$ were female. The median number of medications at the time of admission was seven (IQR 4.25-10).

\section{Length of stay}

The median length of acute inpatient stay for those transferred to intermediate care was 12 days, whereas for those discharged 


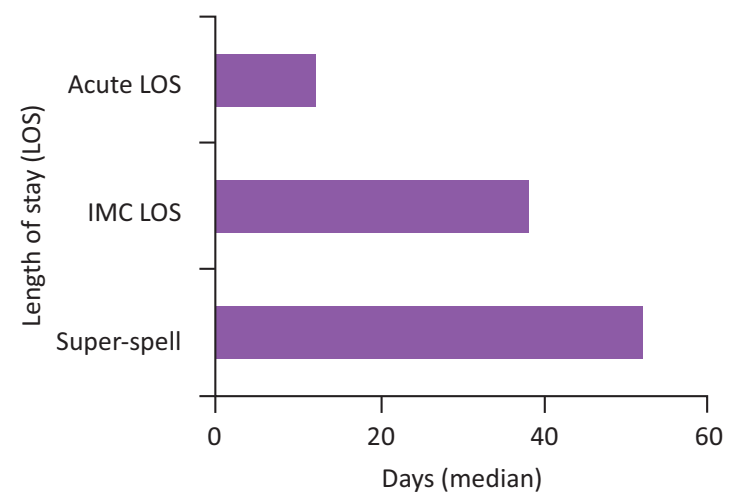

Fig 1. Intermediate care (IMC) cohort: median number of days spent in acute care episode, IMC and super-spell.

directly to the community it was 9.5 days $(p=0.034)$. The median LOS at bedded intermediate care was 38 days (IQR 24.25-58). This figure includes acute LOS for those who were readmitted to hospital and discharged back to intermediate care. The overall combined LOS for those discharged to intermediate care ('superspell') was 52 days (IQR 36.25-72.75), as shown in Fig 1. There was no relationship between acute inpatient LOS and intermediate care LOS.

\section{Delayed transfers of care}

The median wait from being deemed medically fit for discharge to eventual discharge to intermediate care was 3 days (IQR $2-7)$. Thirty-two percent $(n=24)$ of such patients waited $\geq 7$ days as inpatients from being deemed medically fit for discharge to transferring to intermediate care. Delayed transfers of care had no association with mortality or readmissions.

\section{Readmission rates}

There was no significant difference in 30-day readmission rates in those discharged directly home vs IMC. Readmission rate at 120 days was significantly higher in those who were discharged to IMC (OR 3.21, 95\% CI 1.37-7.54, $p=0.0073$ ).

Twenty-six percent of the IMC cohort was readmitted to hospital directly from IMC $(n=20)$. The median number of days from discharge to readmission from IMC was 9 (IQR 3.5-27). The mean acute inpatient LOS following an admission from IMC was 17 days (SD 14.6). Of those that were readmitted, $19.7 \%(n=15)$ of the total IMC cohort was readmitted once and $6.6 \%(n=5)$ were readmitted from IMC twice. The readmission reasons were predominantly medical $(17.1 \%, \mathrm{n}=13)$, followed by orthopaedic $(5.3 \%, n=4)$ and other surgical $3.9 \%(n=3)$.

\section{Mortality}

Only one patient died within 30 days of discharge from hospital; therefore, no significant 30-day mortality difference was recorded between the IMC cohort vs the home cohort. There was no significant difference demonstrated in 120-day mortality between groups $(7.9 \%[n=6]$ in IMC cohort and $1.3 \%[n=1]$ in the home cohort, $p=0.054)$. However, this probably results from a small sample size with the result suggesting a trend to higher mortality in the IMC group.

\section{Associations with cognitive impairment}

Delirium was diagnosed in $25.6 \%$ of all patients $(n=40)$ during their acute inpatient admission. Sixteen percent of patients $(n=25)$ had a pre-existing formal diagnosis of dementia prior to admission.

Patients who received a diagnosis of delirium during their inpatient admission were significantly more likely to be transferred to intermediate care (OR 5.43, 95\% CI 2.36-12.47, $p=0.0001)$. Forty percent of all people who transferred to intermediate care had received a diagnosis of delirium during their acute stay $(n=31)$.

A pre-existing diagnosis of dementia did not have a significant relationship with transfer to intermediate care vs discharge home ( $21.1 \%$ vs $11.3 \%, p=0.147$ )

With regard to ultimate discharge destination following the intermediate care stay, those who had received an acute inpatient diagnosis of delirium were significantly less likely to be discharged to their own home (OR 6.40, 95\% CI 2.25-18.21, $p=0.0005$ ) as were those with a pre-existing diagnosis of dementia (OR 6.60, 95\% CI 1.97-22.08, $p=0.0022$ ).

With regard to the relationship of cognitive impairment with readmission rates, those with delirium were significantly more likely to be readmitted from both the 'home' cohort and the intermediate care cohort at 30 days (OR 2.67, 95\% CI 1.14-2.25, $\mathrm{p}=0.024$ ). A diagnosis of delirium had no significant association with 120 -day readmission rates.

Those with a diagnosis of dementia were significantly more likely to be readmitted from the entire cohort at 120 days (OR $4.7695 \%$ CI 1.79-12.63, $p=0.0017$ ). Dementia diagnosis had no association with 30-day readmission rates.

Diagnoses of delirium or dementia did not have any association with readmission direct from intermediate care.

\section{Associations with Abbreviated Mental Test (AMT) scores}

Thirty-six (23.4\%) had a preoperative AMT score $<8$ while $118(76.6 \%)$ scored eight or more. Fifty-four (35.1\%) had a postoperative score $<8(35.1 \%)$ and $100(64.9 \%)$ had a postoperative score $<8$. Those with low preoperative scores had higher risk of 120-day readmission. A score of less than eight postoperatively was associated with a five times higher risk of a delirium diagnosis. Low AMTs both pre- and postoperatively were associated with longer LOS and 'super-spell'. Low preoperative AMT was associated with longer waits to transfer to IMC (see Table 2). There was no association between AMT scores and transfer to IMC vs discharge home.

\section{Conclusions}

This is the first study comparing outcomes in a hip fracture population attending community bedded intermediate care with those discharged home in an integrated health and social care environment.

The median IMC LOS of 38 days following hip fracture in this study seems, ostensibly, to be considerably longer than the national quoted average of 18 days for hip fracture patients and 
Table 1. Comparisons between those discharged to IMC vs directly home

\begin{tabular}{|c|c|c|c|c|}
\hline & Total $(n=156)$ & Discharged to IMC $(n=76)$ & Discharged home $(n=80)$ & p-value \\
\hline Age (mean) & 80 (SD 8.9) & 83.5 (SD 7.9) & 77.3 (SD 8.8) & .000 \\
\hline Female & $71.2 \%(111)$ & $75 \%(57)$ & $67.5 \%(54)$ & .301 \\
\hline Male & $28.8 \%(45)$ & 25 (19) & $32.5(26)$ & .301 \\
\hline Medications on admission & 7 (IQR 4.25-10) & $8(5.25-11)$ & $6.5(4-9)$ & .007 \\
\hline Inpatient LOS & 11 (IQR 7-18) & 12 days (IQR 8-19) & 9.5 days (IQR 7-15) & .034 \\
\hline 30-day readmission & $17.9 \%(n=28)$ & $18.4 \%(n=14)$ & $17.5 \%(n=14)$ & 1.0 \\
\hline 120-day readmission & $37.8 \%(n=59)$ & $47.3 \%(n=36)$ & $28.8 \%(n=23)$ & .007 \\
\hline Median days to readmission & $33(12-55)$ & $36(12.5-54.25)$ & 22 days (IQR 6-60) & .465 \\
\hline 30-day mortality & $0.6 \%(n=1)$ & $1.3 \%(n=1)$ & 0 & - \\
\hline 120-day mortality & $4.5 \%(n=8)$ & $9.1 \%(n=7)$ & $1.3 \%(n=1)$ & .054 \\
\hline Median days to mortality & $70.5(35.3-98)$ & $80(n=7)$ & 0 & - \\
\hline Dementia diagnosis & $16 \%(25)$ & $21.1 \%(16)$ & $11.3 \%(9)$ & .147 \\
\hline Delirium during admission & $25.6 \%(40)$ & $40.8 \%(31)$ & $11.3 \%(9)$ & .000 \\
\hline
\end{tabular}

26.8 days for all rehabilitation patients. ${ }^{1,5}$ However, the NHFD acknowledges wide variation and challenges in collecting these data with accuracy. Moreover, this is the first data capturing true IMC LOS inclusive of readmissions to hospital within what we view as a continuous care episode.

In the same vein, the median 'super-spell' (acute LOS combined with IMC LOS) of 52 days found in this study is notably longer than the nationally quoted 22.7 days for hip fracture patients. We propose that this study's super-spell duration is more accurate than previous data, which have been acknowledged to be variable and inconsistently recorded. The importance of accurate recording of super-spells across all trusts cannot be overstated in its role of informing decisions regarding commissioning of local services.

These are the first available data relating to delayed transfers of care in this specific group of patients. The median waiting time of
3 days for access to a bed is in keeping with national figures in the general bedded intermediate care population. ${ }^{5}$ Almost one-third of medically fit patients wait for a week or more to be transferred to IMC. Delayed transfers of care represent a significant cost for the healthcare economy by unnecessarily prolonging acute hospital LOS. Further review is required to assess reasons for delayed transfers of care but is likely to be due to multifactorial systemic delays throughout the NHS, not least the consistently low capacity of IMC beds in an era of rising demand for such beds.

Given that a wait of more than 2 days for an intermediate care bed is thought to negate the additional benefit of intermediate care, and a wait of 7 days is associated with a $10 \%$ decline in muscle strength, delayed transfers of care are counterproductive and potentially harmful. ${ }^{5}$ This study highlights the prevalence of such delayed transfers.

Table 2. Associations of pre- and postoperative AMT scores with outcomes

\begin{tabular}{|c|c|c|c|c|c|c|}
\hline & AMT $<8$ preop & AMT $\geq 8$ preop & Sig. & $A M T<8$ postop & $\begin{array}{l}\mathrm{AMT} \geq 8 \\
\text { postop }\end{array}$ & Sig. \\
\hline 30-day and 4-month mortality & $2(1.3 \%)$ & $6(3.9 \%)$ & 1.0 & $4 / 54(7.4 \%)$ & $4 / 100(4 \%)$ & .597 \\
\hline 30-day readmission & $8 / 36(22.2 \%)$ & $19 / 118(16.1 \%)$ & .552 & $14 / 54(25.9 \%)$ & $13 / 100(13 \%)$ & .073 \\
\hline 120-day readmission & $14 / 36(39 \%)$ & $17 / 118(14.4 \%)$ & $\begin{array}{l}.003 \text { OR } 2.7 \\
(1.48-4.9)\end{array}$ & $16 / 54(29.6 \%)$ & $15 / 100(15 \%)$ & .051 \\
\hline Delirium diagnosis & $18 / 36(50 \%)$ & $22 / 118(18.6 \%)$ & $\begin{array}{l}.000 \text { OR } 2.68 \\
(1.6-4.4)\end{array}$ & $30 / 54(55.6 \%)$ & $10 / 100(10 \%)$ & $\begin{array}{l}.000 \text { OR } 5.5 \\
(2.9-10.5)\end{array}$ \\
\hline Pre-existing dementia diagnosis & $18 / 36(50 \%)$ & $7 / 118(5.9 \%)$ & $\begin{array}{l}.000 \text { OR } 8.43 \\
(3.8-18.6)\end{array}$ & $20 / 54(37 \%)$ & $5 \%(5 / 100)$ & $\begin{array}{l}.000 \text { OR } 7.4 \\
(2.9-18.6)\end{array}$ \\
\hline Days awaiting transfer to IMC & $6.5(2.75-9.75)$ & $2(1.5-6.5)$ & $.015 z=-2.43$ & $5(2-8.75)$ & $3(1.5-6.5)$ & .193 \\
\hline Median acute LOS (days) & $17.5(12-26)$ & $9(6.75-14)$ & $.000 z=-4.84$ & $16.5(11-26.75)$ & $9(6-12.75)$ & $\begin{array}{l}.000 \\
z=-5.71\end{array}$ \\
\hline Median super-spell (days) & $62.5(46.75-83)$ & $45(34-70.5)$ & $.032 z=-2.1$ & $64(46.5-81.2)$ & $43(30.5-65)$ & $\begin{array}{l}.003 \\
z=-2.97\end{array}$ \\
\hline
\end{tabular}


Delays in transfers of care may be a barrier to referral to rehabilitation facilities. Future studies could look at attitudes and practices of physicians who make IMC referrals, in relation to local waiting times.

This study is likely to underestimate delayed transfers of care as inevitably some patients are discharged home directly from hospital if they end up meeting their rehabilitation targets as inpatients.

More than a quarter of patients admitted to IMC in this study were readmitted to an acute hospital directly from IMC. This is in contrast with national readmission rates of $10 \%$, which have been quoted in the general bedded intermediate care population. ${ }^{5}$ This discrepancy may be due in part to the heterogeneity of the general rehabilitation population compared with the hip fracture cohort.

Those discharged to IMC are at significantly higher risk of readmission at 120 days compared with those discharged home which is not consistent with recently published research on a large Australian population-based study of veterans and war widows, which followed patients over 1 year. However, that particular study looked at patients in hospital-based IMC which may account for differences in findings. ${ }^{20}$ We postulate that the readmission rate is higher in the IMC group as they are a self-selecting group of frailer, more vulnerable adults than those who are in a position to be discharged home directly from hospital.

As 'short-term' readmission rates are higher in the group with delirium and 'medium-term' readmission rates are higher in those with a pre-existing diagnosis of dementia, perhaps there is a role for investigating targeted intervention in these groups following discharge in order to avoid readmission.

Thirty day mortality across both groups in this study was lower than the national adjusted average of $7.3 \%$. Although there was a trend towards higher 120-day mortality rate in the IMC group, it was not statistically significant and was inadequately powered to definitively demonstrate a difference.

Patients who received a diagnosis of delirium during their inpatient admission were significantly more likely to be transferred to intermediate care than discharged directly home, and are subsequently less likely to be finally discharged home. As detailed assessment of the EPR was used in this study to record delirium, the reported incident rate of delirium is likely to be more accurate than the larger database studies, which reported unusually low rates. The 4AT score has now been introduced to the dataset for use in future reports which should allow for greater accuracy in reporting prevalence of cognitive impairment nationally, and consequently produce larger pieces of data for this important group.

Those with a pre-existing diagnosis of dementia prior to admission were less likely to be discharged home from IMC; this is in keeping with NHFD data which find that those with lower AMT scores (as a crude measure) are less likely go home as their final discharge destination. Contrary to previous research, patients with dementia in this population did not have significantly less rates of access to intermediate care beds. ${ }^{12}$

Prevalence of dementia and incidence of delirium are expected to continue rising in coming years. ${ }^{21}$ Perhaps there is a role for developing dementia- and delirium-friendly IMC facilities. Specialist mental health roles are commissioned specifically within integrated services in $24 \%$ of bed-based intermediate care services nationally at present; ${ }^{5}$ this study suggests a greater need for such roles across the board. Evidence is currently lacking for the best specific approaches to benefit patients with cognitive impairment in order to improve outcomes. ${ }^{22}$ The benefit of rehabilitation in this group has been studied; one study evaluating rehabilitation in patients with dementia following hip fracture found that both inpatient and home-based rehabilitation were associated with lower mortality risk and lower likelihood of admission to 24 -hour care ${ }^{11}$ and several studies have demonstrated functional benefit from rehabilitation in this group. $^{12-14}$

The small sample size of this study prevents accurate interpretation of mortality differences in particular.

Patients who were normally resident in the neighbouring Trafford region were not included in this study as we could not capture the relevant data due to differences in health record systems. Salford council authority is relatively more deprived than Trafford based on social deprivation indices, falling into the top 10 most deprived authorities in England. As this study is based on patients from a single local authority, the results may not be fully generalisable.

However, to its advantage it demonstrates a unique insight into outcomes in an integrated health and social care model, which will be of benefit in informing future service development. This study did not capture outcomes in those with underlying undiagnosed cognitive impairment and relied on previously coded diagnoses of dementia prior to hospital admission. Although resolution of delirium was not a prerequisite to transfer, the delayed transfers of care may have been partially lengthened by waiting for a small number of severe delirium episodes to settle during the acute phase. The 'medically fit for discharge' date is invariably a subjective measure; however, it is documented with relative accuracy in this department.

This study may pave the way for identifying interventions for those who have been found to be at risk for readmission. It also gives useful information regarding associations of cognitive impairment with IMC admissions, acute hospital readmissions, and may guide IMC services to become orientated towards the needs of these individuals.

The information regarding outcomes in those with delirium and dementia is additionally likely to be useful for prognosticating and may inform discussions with patients and families. This study measures outcomes that have not been investigated previously in a UK bedded intermediate care population with hip fracture, including comparing readmission and mortality rates between both groups and quantifying the duration of the continuous care episode in the intermediate care cohort, inclusive of readmissions to hospital.

It should aid future service planning and reinforces the principle that a whole pathway approach to commissioning hip fracture services is imperative.

\section{References}

1 Falls and Fragility Fracture Audit Programme (FFFAP) National Hip Fracture Database annual report 2016. London: RCP, 2016.

2 NICE Clinical Guideline 124: National costing report: Hip fracture. NICE, 2011.

3 Falls and Fragility Fracture Audit Programme (FFFAP) National Hip Fracture Database Annual report 2014. London: RCP, 2014.

4 National Audit Office. Discharging older patients from hospital. London: NAO, 2016. www.nao.org.uk/report/discharging-olderpatients-from-hospital/ [Accessed September 2016]. 
5 National Audit of Intermediate care (NAIC 2015) summary report. NHS Benchmarking, 2015.

6 Seitz DP, Adunuri N, Gill SS, Rochon PA. Prevalence of dementia and cognitive impairment among older adults with hip fractures. J Am Med Dir Assoc 2011;12:556-64.

7 Pioli G, Davoli ML, Pellicciotti F, Pignedoli P, Ferrari A. Comprehensive care. Eur J Phys Rehabil Med 2011:47:265-79.

8 Heruti RJ, Lusky A, Barell V, Ohry A, Adunsky A. Cognitive status at admission: does it affect the rehabilitation outcome of elderly patients with hip fracture? Arch Phys Med Rehabil 1999;80:432-6.

9 Benedetti MG, Ginex V, Mariani E et al. Cognitive impairment is a negative short-term and long-term prognostic factor in elderly patients with hip fracture. Eur J Phys Rehabil Med 2015;51:815-23.

10 Seitz DP, Gill SS, Gruneir A et al. Effects of dementia on postoperative outcomes of older adults with hip fractures: a populationbased study. J Am Med Dir Assoc 2014;15:334-41.

11 Seitz DP, Gill SS, Austin PC et al. Rehabilitation of Older Adults with Dementia After Hip Fracture. J Am Geriatr Soc 2016;64:47-54.

12 Mitchell R, Harvey L, Brodaty H, Draper B, Close J. Hip fracture and the influence of dementia on health outcomes and access to hospital-based rehabilitation for older individuals. Disabil Rehabil 2016;38:2286-95.

13 Resnick B, Beaupre L, McGilton KS et al. Rehabilitation interventions for older individuals with cognitive impairment post hip fracture: a systematic review. J Am Med Dir Assoc 2016;17:200-5.

14 McGilton KS, Chu CH, Naglie G et al. Factors influencing outcomes of older adults after undergoing rehabilitation for hip fracture. J Am Geriatr Soc 2016:64:1601-9.

15 Gustafson Y, Berggren D, Brännström B et al. Acute confusional states in elderly patients treated for femoral neck fracture. J Am Geriatr Soc 1988;36:525-30.
16 Marcantonio ER, Flacker JM, Michaels M, Resnick NM. Delirium is independently associated with poor functional recovery after hip fracture. J Am Geriatr Soc 2000;48:618-24.

17 Dubljanin-Raspopovic E, Markovic-Denic L, Mrinkovic ] et al. Use of early indicators in rehabilitation process to predict one-year mortality in elderly hip fracture patients. Hip Int 2012;22:661-7.

18 Radinovic K, Markovic-Denic L, Dubljanin-Raspopovic et al. Estimating the effect of incident delirium on short-term outcomes in aged hip fracture patients through propensity score analysis. Geriatr Gerontol Int 2015;15:848-55.

19 Krogseth M, Wyller TB, Engedal K, Juliebo V. Delirium is a risk factor for institutionalisation and functional decline in older hip fracture patients. J Psychosom Res 2014;76:68-74.

20 Ireland AW, Kelly PJ, Cumming RG. Associations between hospitalbased rehabilitation for hip fracture and two-year outcomes for mortality and independent living: An Australian database study of 1,724 elderly community-dwelling patients. J Rehabil Med 2016:48:625-31.

21 Adunsky A, Levy R, Heim M, Mizrahi E, Arad M. The unfavourable nature of preoperative delirium in elderly hip fractured patients. Arch Gerontol Geriatr 2003:36:67-74.

22 Smith TO, Hameed YA, Cross JL et al. Enhanced rehabilitation and care models for adults with dementia following hip fracture surgery. Cochrane Database Syst Rev 2015;15:CD010569.

Address for correspondence: Dr Una Clancy, Western General Hospital, Crewe Road South, Edinburgh EH4 2XU, UK.

Email: una.clancy@nhslothian.scot.nhs.uk

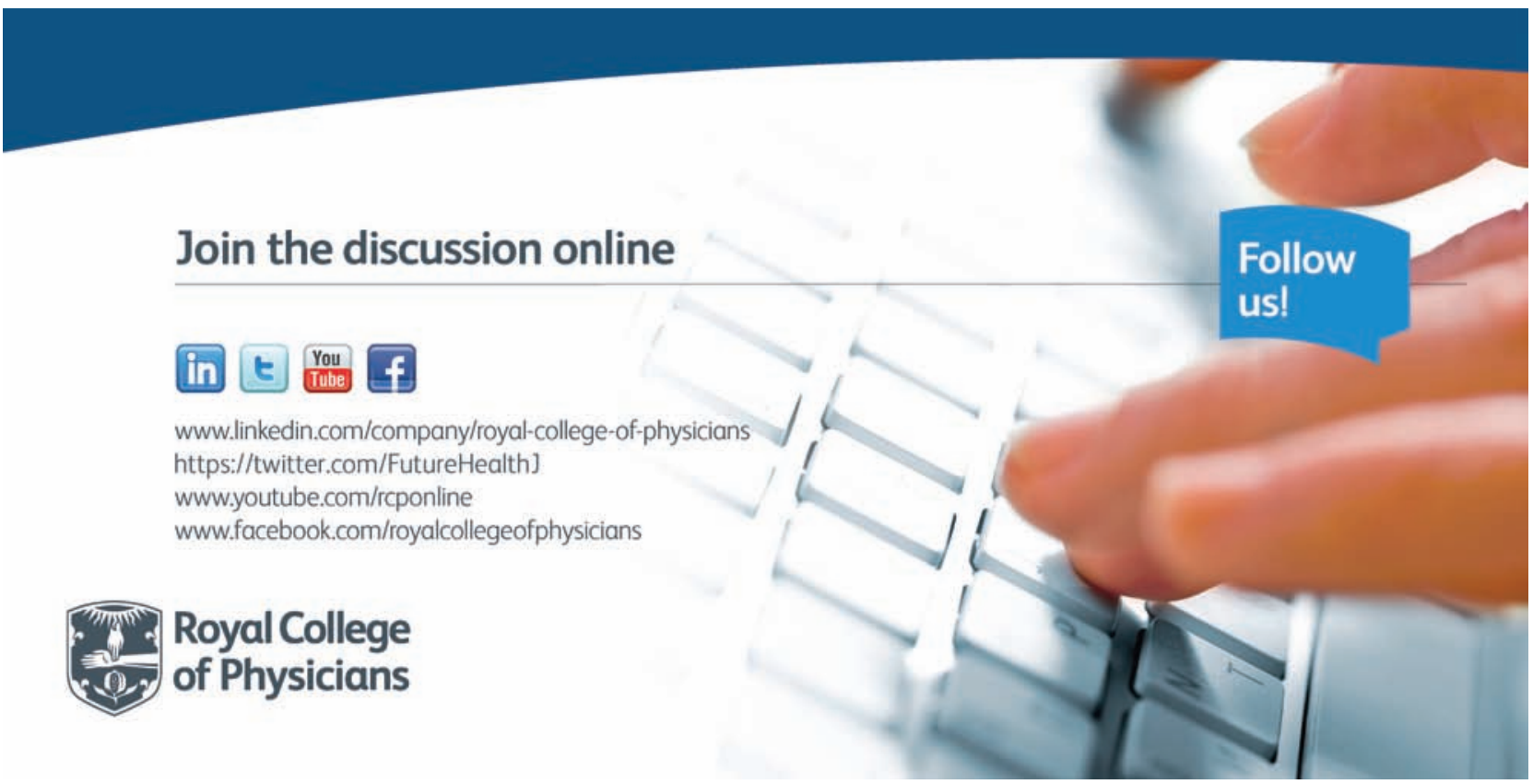

